\title{
Pleurectomy/decortication, hyperthermic pleural lavage with povidone-iodine, prophylactic radiotherapy, and systemic chemotherapy in patients with malignant pleural mesothelioma: A 10-year experience
}

\author{
Loïc Lang-Lazdunski, MD, PhD, ${ }^{\mathrm{a}, \mathrm{c}}$ Andrea Bille, MD, ${ }^{\mathrm{a}}$ Sophie Papa, MD, ${ }^{\mathrm{b}}$ Stuart Marshall, FRCA, ${ }^{\mathrm{a}}$ \\ Rohit Lal, PhD, ${ }^{\mathrm{b}}$ Carlotta Galeone, ScD, PhD, ${ }^{\mathrm{e}}$ David Landau, FRCR, ${ }^{\mathrm{b}}$ Jeremy Steele, MD, FRCP, ${ }^{\mathrm{d}}$ and \\ James Spicer, PhD, FRCP ${ }^{\mathrm{b}, \mathrm{c}}$
}

\begin{abstract}
Objectives: We evaluated the long-term results of pleurectomy/decortication (P/D), hyperthermic pleural lavage with povidone-iodine, prophylactic chest wall radiotherapy (21 Gy), and systemic chemotherapy in patients with malignant pleural mesothelioma.
\end{abstract}

\begin{abstract}
Methods: A cohort of patients having surgery between January 2004 and December 2013 were retrospectively studied. All patients received prophylactic radiotherapy postoperatively and all were supposed to receive systemic chemotherapy, either preoperatively or as adjuvant therapy. Patients were reviewed at 30 days, then followed up 6-monthly. ${ }^{18}$ F-FDG-PET-CT was used routinely to diagnose disease recurrence. Second-line therapies were administered when appropriate. Survival and prognostic factors were analyzed by the Kaplan-Meier method, log-rank test, and Cox regression analysis.
\end{abstract}

Results: One hundred two patients had P/D followed by prophylactic radiotherapy and were referred for adjuvant chemotherapy. Median age at operation was 64 years. Eighty-one patients $(79.4 \%)$ were male; 57 patients $(55.9 \%)$ had complete macroscopic resection. Thirty-day mortality was nil and 30 patients $(29.4 \%)$ experienced postoperative complications. Seventy-three patients had epithelioid mesothelioma (71.5\%). Sixty-eight patients $(66.6 \%)$ had N0 disease. Ninety-six patients $(94.1 \%)$ received the planned 4 to 6 chemotherapy cycles. At last follow-up, 49 patients were alive. Univariate analysis showed no significant difference when sex, age $>70$ years, nodal status, or prior chemotherapy were considered. The overall median survival was 32 months and 5-year survival rate was $23.1 \%$. Median survival and 5-year survival rates were 35.0 months and $30.7 \%$ for epithelioid mesothelioma and 15 months and $7 \%$ for nonepithelioid mesothelioma, respectively $(P=.0001)$. Median survival was 45.0 months for R0-R1 resection versus 17.4 months for $\mathrm{R} 2$ resection $(P=.0001)$.

Conclusions: P/D, hyperthermic pleural lavage with povidone-iodine, prophylactic chest wall radiotherapy, and systemic chemotherapy is a safe and well-tolerated multimodality therapy. (J Thorac Cardiovasc Surg 2015;149:558-66)

See related commentary on pages 567-8.

From the Departments of Thoracic Surgery ${ }^{\mathrm{a}}$ and Oncology, ${ }^{\mathrm{b}}$ Guy's \& St Thomas Hospital, London, United Kingdom; Division of Cancer Studies, ${ }^{c}$ King's College London, London, United Kingdom; Department of Medical Oncology, ${ }^{\mathrm{d}}$ Lung and Mesothelioma Unit, St Bartholomew's Hospital, London, United Kingdom; and Department of Clinical Sciences and Community Health, ${ }^{\mathrm{e}}$ University of Milan, Milan, Italy.

Disclosures: James Spicer reports consulting fees for Boehringer Ingelheim and Roche. Rohit Lal reports lecture fees for Boehringer Ingelheim. All other authors have nothing to disclose with regard to commercial support.

Read at the 94th Annual Meeting of The American Association for Thoracic Surgery, Toronto, Ontario, Canada, April 26-30, 2014.

Received for publication March 30, 2014; revisions received Sept 14, 2014; accepted for publication Oct 6, 2014

Address for reprints: Loïc Lang-Lazdunski, MD, PhD, Department of Thoracic Surgery, Guy's Hospital, Great Maze Pond, London SE1 9RT, United Kingdom (E-mail: loic.lang-lazdunski@gstt.nhs.uk).

$0022-5223 / \$ 36.00$

Copyright (c) 2015 by The American Association for Thoracic Surgery

http://dx.doi.org/10.1016/j.jtcvs.2014.10.041
Malignant pleural mesothelioma (MPM) is a fatal malignancy linked to asbestos exposure for which there is no known cure. ${ }^{1}$ The incidence of mesothelioma has increased continuously over the past 20 years and is expected to peak around 2020 in Western Europe. ${ }^{1}$ At present, the only standard treatment for MPM is palliative chemotherapy. ${ }^{2}$ Median survival following diagnosis is often less than 12 months., ${ }^{2,3}$ Patients with early-stage disease and good performance status are suitable for multimodality therapy involving surgery, radiotherapy, and chemotherapy. ${ }^{4}$ However, such treatment is only feasible in well patients without significant comorbidities. ${ }^{5}$ Multimodality therapy has evolved over the years. Extrapleural pneumonectomy (EPP) gained wide acceptance following publications showing prolonged survival in patients with favorable prognostic factors. ${ }^{5-9}$ Although the 30-day mortality of EPP is presently less than 5\% at most large-volume institutions, less than $50 \%$ of patients complete the trimodality 

Abbreviations and Acronyms
$\mathrm{CT}=$ computed tomography
$\mathrm{EPP}=$ extrapleural pneumonectomy
$\mathrm{MPM}=$ malignant pleural mesothelioma
$\mathrm{P} / \mathrm{D}=$ pleurectomy/decortication
PET = positron emission tomography
PTFE $=$ polytetrafluoroethylene
$\mathrm{TNM}=$ tumor-node-metastasis

treatment because of death or major complications. ${ }^{7-12}$ Moreover, when patients relapse following EPP, they usually die within a few months. ${ }^{13}$

Pleurectomy/decortication (P/D) has long been used in patients with MPM with or without radiotherapy and chemotherapy. ${ }^{14-19}$ In 2008, Flores et al reported a large experience acquired at 3 different institutions, on 663 patients. They found that patients undergoing P/D had a better survival than those undergoing EPP but noticed that the reasons were multifactorial and subject to selection bias. ${ }^{20}$ Since 2004, we have offered EPP and P/D as part of our multimodality therapy program. A significant proportion of our patients were not suitable for EPP or refused EPP, and we have therefore developed an alternative multimodality therapy plan for these patients based on P/D and hyperthermic pleural lavage with povidone-iodine followed by prophylactic radiotherapy. Systemic chemotherapy was offered preoperatively or as an adjuvant treatment. This article presents our 10-year experience with this multimodality regimen.

\section{PATIENTS AND METHODS}

Since January 2004, all patients having P/D and hyperthermic pleural lavage with povidone-iodine followed by prophylactic radiotherapy at our institution have been studied retrospectively and entered into a database. Patients deemed suitable for multimodality therapy were offered P/D upfront followed by prophylactic radiotherapy at 4 to 5 weeks, and adjuvant chemotherapy started at 6 to 8 weeks, postoperatively. We did also accept patients for P/D after neoadjuvant/induction chemotherapy, when they showed response to chemotherapy. Surgical protocols were approved by the institutional review board, and informed consent was obtained from each patient before each treatment step. Recruitment was via a specialized multidisciplinary thoracic oncology clinic involving thoracic surgeons, radiation oncologists, and medical oncologists. Histopathology and imaging review was undertaken in all patients. The choice of multimodality treatment was determined by patient's performance status, comorbidities, clinical tumor-node-metastasis (TNM) stage, histological subtype, and patient's preference. All patients received oral and written information about mesothelioma treatments and were offered several appointments to discuss their treatment plan. All patients had chest computed tomography (CT) and ${ }^{18} \mathrm{~F}$-fluorodeoxyglucose-positron emission tomography (PET)CT performed before surgery to exclude N3 disease or extrathoracic metastases. Previous talc pleurodesis was not regarded as a contraindication to the performance of P/D. Nonbulky N2 disease was not regarded as a contraindication to $\mathrm{P} / \mathrm{D}$ in this study either. Only patients with performance status 0 to 1 were offered multimodality therapy and patients with pejorative prognostic factors such as anemia, thrombocythemia, high white blood cell count, and weight loss were excluded and referred for palliative chemotherapy. All patients had a pulmonary function test (spirometry and gas transfer study), and those with a history of angina had a stress echocardiogram plus or minus coronary angiography to rule out significant coronary artery disease. All patients having P/D performed were entered into our database.

\section{Surgical Protocol}

Patients received an epidural catheter prior to commencement of the procedure. All patients had invasive arterial blood pressure and central venous pressure monitoring, operated on with a double-lumen tracheal tube, a nasogastric tube, and placed in the appropriate lateral decubitus position with Ted stockings and pneumatic boots on throughout the operation. All patients had pulse oximetry monitored continuously and received prophylactic antibiotics (cefuroxime) on induction. All patients were monitored overnight in a high-dependency unit and returned to the thoracic surgical ward on day 1 or later if appropriate. Deep vein thrombosis prophylaxis was started the night before surgery and continued for 30 days postoperatively (enoxaparin $40 \mathrm{mg}$ daily, subcutaneously).

The goal in all patients was to achieve maximal cytoreduction and a complete macroscopic resection of the tumor (R0 or R1). The approach was always a posterolateral thoracotomy through the fifth intercostal space. A partial rib resection (sixth) was used in patients with contracted hemithorax. In some patients, a second access thoracotomy was made in the eighth interspace to help achieve complete macroscopic resection of tumor in the diaphragmatic recess or to resect and graft the diaphragm. Total pleurectomy and decortication was performed in all patients. Extended resection to include the pericardium, lung parenchyma, and diaphragm was undertaken as necessary. When the diaphragm was involved (deep muscular invasion), it was resected and reconstructed using a large polytetrafluoroethylene (PTFE) mesh (2-mm Dual Mesh, Gore-Tex, W. L. Gore \& Associates UK Ltd, Livingston, West Lothian, United Kingdom). Mediastinal lymphadenectomy was routinely performed. Anterior mediastinal fat was excised en bloc with pleural specimens.

Once the P/D had been completed and the lung reexpanded to check for air leaks, we performed a hyperthermic pleural lavage $\left(40^{\circ} \mathrm{C}-41^{\circ} \mathrm{C}\right)$ using sterile water mixed with $10 \%$ povidone-iodine (dilution $1 / 10$, Videne; Ecolab. Ltd, Gartforth, Leeds, United Kingdom). In all patients, 5 to $6 \mathrm{~L}$ of water was used. The washout was allowed to bath in the pleural cavity for 5 minutes while the lung was collapsed and was then aspirated slowly. This maneuver was repeated 3 times. The thoracotomy wound was irrigated with pure $10 \%$ povidone-iodine before closure. Phrenic nerve block $(0.5 \%$ Marcaine [bupivacaine $\mathrm{HCl}$ ], $5 \mathrm{~mL}$; AstraZeneca UK Ltd, Luton, United Kingdom) was routinely done to prevent shoulder pain. Two to 3 drains (28-F) were placed in the chest cavity before closing. The extent of resection and clinical TNM stage was recorded by the operator.

\section{Prophylactic Radiotherapy Protocol}

All patients were reviewed in the outpatient clinic at 4 weeks postoperatively and referred for prophylactic radiotherapy ( $21 \mathrm{~Gy}$ in 3 daily fractions) on the thoracotomy, drain, and previous port sites according to the Boutin protocol. ${ }^{21}$

\section{Preoperative Chemotherapy Protocol}

All patients had received platinum-based chemotherapy completed less than 6 weeks before surgery. The regimen consisted of a combination of pemetrexed and cisplatin. All patients had received 4 or 6 cycles, preoperatively. Patients were referred either after completing 6 cycles of chemotherapy at another institution or after completing 4 cycles of induction chemotherapy at our institution and having repeat imaging showing an objective response.

\section{Adjuvant Chemotherapy Protocol}

Adjuvant chemotherapy was started 6 to 8 weeks following surgery, when the patient had recovered enough and the wounds had healed 
satisfactorily. The regimen consisted of a combination of gemcitabine and cisplatin before 2007, pemetrexed and cisplatin thereafter. The intent was to administer 4 to 6 cycles of chemotherapy. Dose reduction was allowed, depending on clinical and hematologic tolerance. Patients tolerating chemotherapy well after 4 cycles were allowed to receive 6 cycles. Most patients were treated as outpatients at our institution; others received chemotherapy at their original referral center for practical reasons.

All patients were followed up in the outpatient clinic regularly and had PET-CT or chest CT arranged 6-monthly to monitor response to treatment or disease progression. Those relapsing following multimodality therapy were offered second-line therapies: rechallenge with platinum-based chemotherapy (with pemetrexed, gemcitabine, or irinotecan) or single agent vinorelbine, or targeted therapy as part of a clinical trial. Radiotherapy was offered as a palliative measure when patients were diagnosed with relapse, or as a curative treatment, exceptionally.

\section{Statistical Analysis}

Continuous data are reported with medians and ranges, whereas categorical data are reported with counts and percentages. All potential prognostic indices were measured at the time of operation and evaluated as categorical variables. Survival curves were computed according to the Kaplan-Meier method. Survival was calculated from the date of mesothelioma diagnosis. Survival comparison between groups of patients undergoing $\mathrm{P} / \mathrm{D}$ was performed using the log-rank test. Factors significantly affecting survival were tested in the univariate analysis. Confidence intervals $(95 \%)$ were used to quantify extent of the observed differences. Factors that significantly affected survival in univariate analysis $(P<.10)$ were tested for their independent role in multivariate analysis using the Cox proportional hazards model. The stepwise backward procedure based on the likelihood ratio was used to assess the significance of covariates included in the model. Hazard ratios and $95 \%$ confidence intervals were calculated.

All tests were 2 sided and $P$ values less than .05 were considered statistically significant. Statistical analyses were performed using an SPSS (version 18.0; SPSS Inc, Chicago, Ill) software package and the figures were obtained using Stata statistical software (version 11; StataCorp, College Station, Tex).

\section{RESULTS}

One hundred two patients had P/D performed by a single surgeon (L.L.L.) between January 2004 and December 2013 with the goal of achieving complete macroscopic resection. Four patients had acute empyema at the time of the procedure, secondary to previous talc pleurodesis. A total of 102 patients had P/D followed by prophylactic radiotherapy. Fourteen patients had received chemotherapy (4-6 cycles of pemetrexed and cisplatin) before P/D.

Eighty-one $(79.4 \%)$ patients were male and $21(20.6 \%)$ were female. Median age at operation was 64.7 years (range 32-81 years). Twenty-four patients $(23.5 \%)$ were older than 70 years. Sixty-two procedures $(60.8 \%)$ were right-sided, 40 left-sided. Fifty-seven patients (55.9\%) underwent R0-R1 resection whereas 45 underwent R2 resection (44.1\%). Extended resections included diaphragm $(\mathrm{n}=8)$, lobectomy $(\mathrm{n}=3)$, bilobectomy $(\mathrm{n}=1)$, segmentectomy $(\mathrm{n}=2)$, pericardectomy requiring reconstruction $(\mathrm{n}=3)$, superior vena cava resection $(\mathrm{n}=1)$, and chest wall requiring reconstruction $(\mathrm{n}=1)$.
There was no 30-day mortality and no 90-day mortality. Thirty patients $(29.4 \%)$ experienced postoperative complications (Table 1). Final histopathology showed that 73 patients had epithelioid mesothelioma (71.5\%), 25 had biphasic mesothelioma $(24.5 \%)$, and 4 patients had sarcomatoid mesothelioma (3.9\%).

Sixty-eight patients $(66.6 \%)$ had N0 disease, 8 patients had N1 disease $(7.8 \%)$, and 26 patients had $\mathrm{N} 2$ disease $(25.5 \%)$. Distribution was as follows: pT1N0M0, $\mathrm{n}=7$; pT2NOM0, $\mathrm{n}=24$; pT3NOM0, $\mathrm{n}=28$; pT4NOM0, $\mathrm{n}=9$; pT3N1M0, $\mathrm{n}=7$; pT4N1M0, $\mathrm{n}=1$; $\mathrm{pT} 2 \mathrm{~N} 2 \mathrm{M} 0, \mathrm{n}=8$; pT3N2M0, $\mathrm{n}=15$; $\mathrm{pT} 4 \mathrm{~N} 2 \mathrm{M} 0, \mathrm{n}=3$.

International Mesothelioma Interest Group (IMIG) classification stages were I, $\mathrm{n}=7(6.9 \%)$; II, $\mathrm{n}=24$ $(23.5 \%) ;$ III, $\mathrm{n}=58(56.9 \%)$; and IV, $\mathrm{n}=13(12.7 \%)$, respectively.

Six patients did not start adjuvant chemotherapy within the agreed period of 8 weeks: 4 patients deteriorated rapidly and were not fit to receive chemotherapy. The fifth and sixth patients refused chemotherapy initially but agreed to receive pemetrexed and platinum salt subsequently.

Eighty-three patients received 4 to 6 cycles of chemotherapy (Figure 1). In total, 97 patients $(95 \%)$ received 4 to 6 cycles of chemotherapy either preoperatively $(\mathrm{n}=14)$ or postoperatively $(\mathrm{n}=83)$. Three patients developed late complications after receiving first-line chemotherapy. Two patients reaccumulated fluid in the pleural cavity following P/D and had an indwelling pleural catheter inserted for a few weeks. One patient suffered a pulmonary embolus shortly after his fifth chemotherapy cycle and was anticoagulated. One patient underwent completion EPP at another hospital 10 months after P/D, and another one underwent bi-lobectomy for a massive parenchymal recurrence at 19 months and developed a late bronchopleural fistula. We did not observe wound infections in this series. Patients diagnosed with disease progression at follow-up were routinely offered secondline and eventually third-line therapy. Most second-line therapies involved single agent pemetrexed or pemetrexed combined with platinum $(\mathrm{n}=17)$, gemcitabine and carboplatin $(n=1)$, irinotecan and cisplatin $(n=2)$, or single agent vinorelbine $(n=5)$. Five patients were enrolled in a phase 2 study of single agent sorafenib and 1 patient in a phase 1 study of focal adhesion kinase inhibitor. Four patients enrolled into a randomized phase 2 study of pegylated arginine deaminase (ADAM trial). Two patients diagnosed with focal relapse of tumor at 1 year following P/D were treated with CyberKnife radiosurgery (Accuray Inc, Madison, Wis). One remains disease-free at 80 months postoperatively; the second one has low-volume recurrent disease and remains well at 40 months. Of note, none of our patients was diagnosed with chest wall tumor seeding at follow-up. 
TABLE 1. Postoperative complications

\begin{tabular}{lc}
\hline \multicolumn{1}{c}{ Complication } & n (\%) \\
\hline Persistent air leak $>5 \mathrm{~d}$ & $17(56.6)$ \\
Chylothorax* & $4(13.3)$ \\
Pneumonia & $3(10)$ \\
Empyema & $2(6.6)$ \\
Atrial fibrillation & $2(6.6)$ \\
ARDS & $1(3.3)$ \\
TIA & $1(3.3)$ \\
\hline
\end{tabular}

ARDS, Adult respiratory distress syndrome; TIA, transient ischemic attack. *Patients requiring thoracotomy $(\mathrm{n}=3)$ or video-assisted thoracoscopic surgery $(\mathrm{n}=1)$ to ligate the thoracic duct.

At last follow-up, 49 patients were alive (median followup, 33 months; range, 3-97 months) and 76 patients diagnosed with tumor relapse (Table 2). The mean time to first relapse was $19.6 \pm 18.2$ months (median, 12 months).

Univariate analysis showed no significant difference when sex $(P=.635)$, age $>70$ years $(P=.372)$, side of disease $(P=.617)$, nodal status (N0 vs N1/N2) $(P=.078)$, or prior chemotherapy $(P=.339)$ were considered. The median survival of patients with N0 disease and $\mathrm{N} 1 / \mathrm{N} 2$ disease were 32.3 months (95\% CI, 23.5-40.9) and 30.4 months $(95 \% \mathrm{CI}, 19.3-41.7)$, respectively.

Histology had a significant impact on survival $(P=.0001)$ : patients with epithelioid mesothelioma had a median survival of 35.3 months (95\% CI, 20.4-49.6), whereas those with nonepithelioid mesothelioma had a median survival of 15.0 months (95\% CI, 11.2-18.7). Completeness of resection also had a significant impact on survival, and patients undergoing complete macroscopical resection (R0-R1) had better median survival (45.0 months, 95\% CI, 26.9-63.0) than those who underwent an incomplete macroscopic resection (R2) (17.4 months, 95\% CI, 10.5-24.3) $(P=.0001)$.

Figure 2 shows patients' overall survival. Median overall survival was 32 months (95\% CI, 25.6-38.4). One-, 2-, and

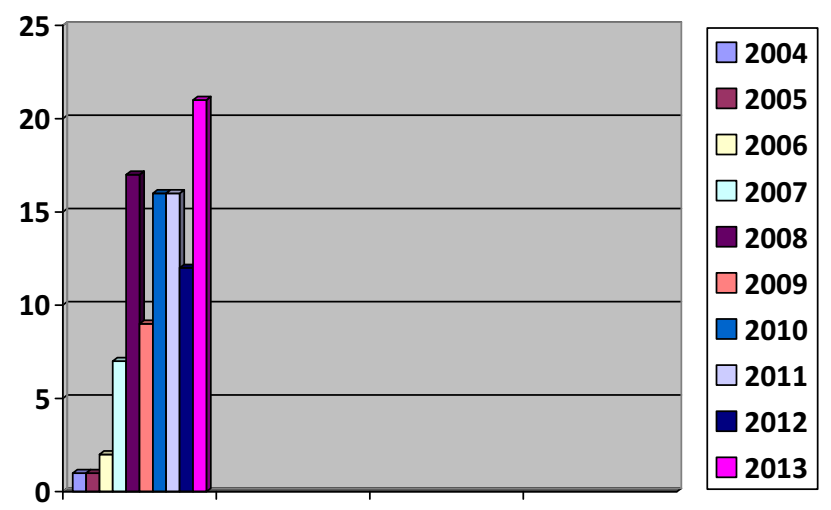

FIGURE 1. Flow chart of patients (2004-2013): 14 had preoperative chemotherapy and 83 adjuvant chemotherapy. All received adjuvant prophylactic radiotherapy.
TABLE 2. Sites of first recurrence in 76 patients

\begin{tabular}{lc}
\hline \multicolumn{1}{c}{ Site } & n (\%) \\
\hline Ipsilateral pleural cavity or lung & $68(89.5)$ \\
Contralateral pleural cavity or lung & $2(2.6)$ \\
Abdomen & $2(2.6)$ \\
Thoracic lymph nodes & $2(2.6)$ \\
Bones & $2(2.6)$ \\
\hline
\end{tabular}

5-year survival rates were $87.2 \%, 62.9 \%$ and $23.1 \%$, respectively.

Figure 3 shows the comparative survival of patients according to histology: for epithelioid mesothelioma, 1-, $2-$, and 5-year survival rates were $94.5 \%, 76.5 \%$, and $30.7 \%$, respectively; for nonepithelioid histology, 1-, 2-, and 5-year survival rates were $69 \%, 31.7 \%$, and $7 \%$, respectively. Figure 4 shows comparative survival according to nodal status: patients with N0 disease had 1-, 2-, and 5-year survival rates of $89.5 \%, 66.7 \%$, and $30 \%$, respectively; those with N1/N2 disease had 1-, 2-, and 5 -year survival rates of $85.3 \%, 58 \%$, and $0 \%$, respectively. Figure 5 shows comparative survival according to completeness of resection: patients with complete macroscopic resection had 1-, 2-, and 5-year survival rates of $98.2 \%, 83.2 \%$, and $34.8 \%$, respectively; those with incomplete macroscopic resection (R2) had 1-, 2-, and 5-year survival rates of $73.3 \%, 35.4 \%$, and $6.9 \%$, respectively.

At multivariate analysis, only epithelioid histology $(P=.0001$, hazard ratio, 4.27; 95\% CI, 2.355-7.743) and complete resection $(P=.0001$, hazard ratio, 3.4; 95\% CI, 1.920-6.110) were 2 independent prognostic factors. Forty-six patients cumulated 2 good prognostic factors (epithelioid histology, and complete macroscopic resection) and had a median survival of 46.4 months (95\% CI, 24.7-68.1). Those patients had 1-, 2-, and 5-year survival rates of $97.8 \%, 87.4 \%$, and $40.2 \%$, respectively.

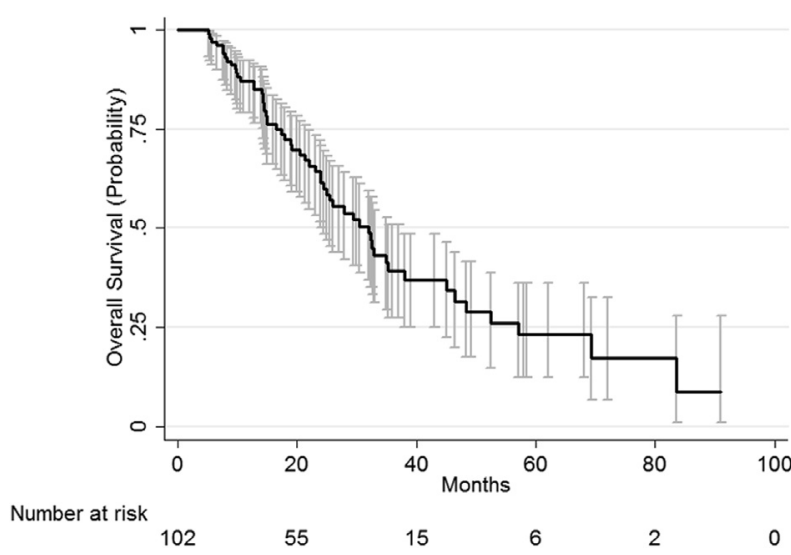

FIGURE 2. Global overall survival, $n=102$ (Kaplan-Meier). 


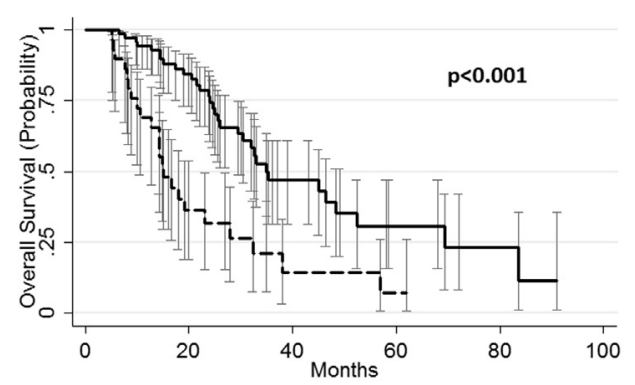

Number at risk

Epithelioid histolc

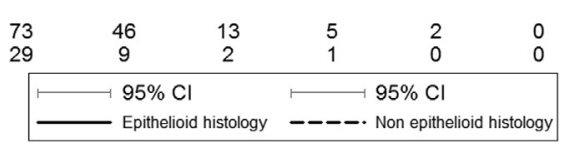

FIGURE 3. Comparative survival according to histology (Kaplan-Meier). $C I$, Confidence interval.

Of note, 4 patients were referred for $P / D$ with a preexisting diagnosis of pleural empyema following talc pleurodesis. P/D was performed with a curative intent in all patients, but complete macroscopic resection could not be achieved in 3 patients. When analyzed separately, those patients were not significantly older (median age 69 years, range 57-74), 2 had epithelioid tumors, and 2 had stage IV sarcomatoid tumors diagnosed at surgery. Median overall survival for those 4 patients was 10.5 months $(5,6,16$, and 26 months, respectively). The only survival beyond 2 years in this subgroup of patients was in the only patient receiving adjuvant chemotherapy.

\section{DISCUSSION}

Five decades after Wagner pointed out the link between asbestos and MPM, there is still no recognized cure for MPM. ${ }^{10}$ Advocates of surgery suggest that multimodality therapy involving EPP provides significantly longer survival than palliative chemotherapy. Recent series showed 5-year survival rates higher than $40 \%$ in highly selected patients demonstrating favorable prognostic

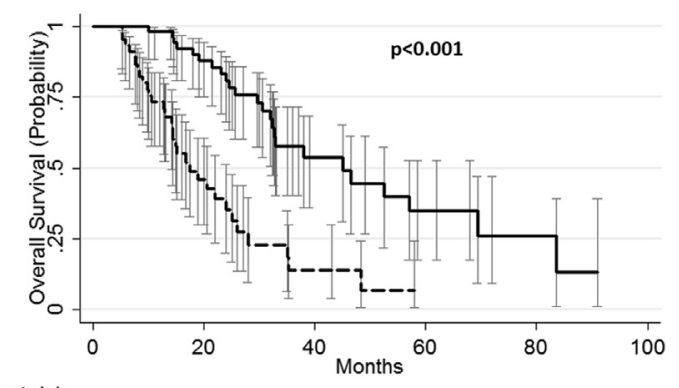

Number at risk

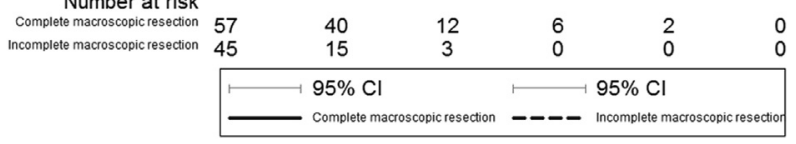

FIGURE 4. Comparative survival according to nodal status (N0 vs N1/ N2) (Kaplan-Meier). CI, Confidence interval.

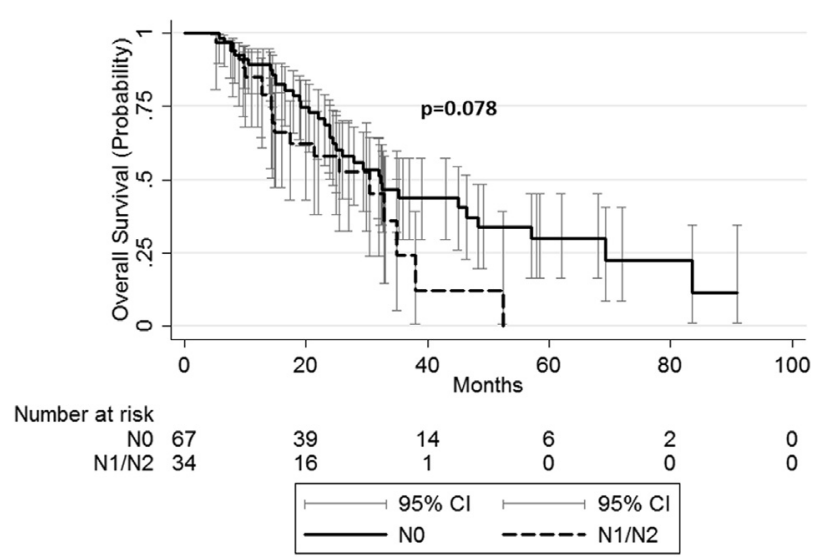

FIGURE 5. Comparative survival according to completeness of resection (R0-R1 vs R2) (Kaplan-Meier). CI, Confidence interval.

factors. ${ }^{5,9}$ In most series, information was acquired retrospectively, as in our study. Of note, in 2 recent prospective studies, less than $55 \%$ of patients could complete the full multimodality treatment plan. ${ }^{7,10}$ EPP is a "maximally invasive" procedure. Although mortality has fallen consistently over time from $33 \%$ to less than $5 \%$ at most specialist centres, ${ }^{8,9,11,22}$ morbidity remains high, with serious complications developing in more than $50 \%$ of patients. ${ }^{11}$ Therefore, only fit patients and those without comorbidities qualify for this operation. When patients have recovered from EPP, radical radiotherapy is administered and this can generate significant complications and mortality as well. ${ }^{12}$ The benefits of EPP appear to be limited to patients with $\mathrm{cN} 0 / \mathrm{N} 1$ disease and epithelioid subtype of mesothelioma. ${ }^{5}$ Lastly, lack of treatment options following disease recurrence after EPP and radiotherapy means that death usually occurs within 3 months following recurrence. ${ }^{13}$

Opponents of radical surgery suggest that some patients survive for several years with palliative chemotherapy only following pleurodesis. There are anecdotal reports of spontaneous regression of MPM or very prolonged survival without aggressive therapy. ${ }^{23}$ At present, the standard of care for those unsuitable for multimodality therapy is palliative chemotherapy with a combination of pemetrexed and cisplatin. ${ }^{3}$ This regime has been associated with a median survival of 12 months in a large randomized trial. ${ }^{3}$ Interestingly, a recent study showed an equivalent median survival of 12.8 months in a selected population of patients receiving multimodality therapy involving EPP and hyperthermic intrapleural chemotherapy. ${ }^{24}$

Historical experiences with P/D showed variable mortality and morbidity, especially when it was combined with intrapleural chemotherapy. ${ }^{14-18}$ In-hospital mortality varied from $3.6 \%$ to $11 \%$ and median overall survival from 13 to 17 months with intrapleural chemotherapy. ${ }^{17,18}$ The addition of adjuvant radiotherapy did not seem to confer 
any survival advantage but was the source of radiation pneumonitis in a large proportion of patients in 2 reports. ${ }^{16,19}$ Recent progress in radiotherapy techniques now allow for the hemithorax to be irradiated but the lung to be spared following P/D. A recent study reported a low rate of grade 3 to 4 pneumonitis after $\mathrm{P} / \mathrm{D}$. $^{25}$ Patterns of relapse following EPP or P/D have been well studied and described. ${ }^{13,26}$ Although patients relapse mostly locally in the chest cavity following $\mathrm{P} / \mathrm{D}$, patients appear to experience disease progression in the abdomen, mediastinum, contralateral lung, and other distant sites following EPP. ${ }^{13}$ We made no attempt to convert to EPP when complete macroscopic resection could not be achieved at $\mathrm{P} / \mathrm{D}$, as we found previously that EPP provided no survival advantage. ${ }^{27}$

Our objective was to develop a surgical strategy suitable for most patients, including those with sarcomatoid histology and those with N2 disease. Patients with sarcomatoid histology have a dismal prognosis no matter which treatment is used, and it is now our opinion that radical surgery should not be offered in this group of patients. As virtually all patients have residual microscopic disease after P/D, it seemed essential to prevent chest wall seeding by administering prophylactic radiotherapy on the thoracotomy and drain wounds according to the Boutin protocol. ${ }^{21}$ Although the role of prophylactic radiotherapy has been controversial, our local protocol involves $21 \mathrm{~Gy}$ in 3 daily fractions following thoracoscopy or videoassisted thoracoscopic surgery. We were quite worried about the possible detrimental effects of high-dose hemithoracic radiotherapy ( $>45 \mathrm{~Gy}$ ) and decided to administer a lower dose to the chest wall to try to prevent secondary malignant implants in the thoracotomy or drain sites. Of note, none of the 102 patients had implants in the thoracotomy or drain sites at follow-up.

We thought it was important to obtain a maximal cytoreduction before administering chemotherapy and used adjuvant rather than neoadjuvant chemotherapy. Thus, several groups have reported on the beneficial effects of cytoreduction (tumor debulking) on the antitumor immune response in animal models of mesothelioma. Robinson and his group showed that partial, but not complete tumor debulking surgery promotes protective antitumor memory when combined with chemotherapy and adjuvant immunotherapy. ${ }^{28}$ More recently, Albelda and his group have showed that cytoreductive surgery reduces systemic myeloid suppressor cell populations and restores intratumoral immunotherapy effectiveness. ${ }^{29}$

Cytoreductive surgery and hyperthermic intraoperative intraperitoneal chemotherapy have shown promising results in large series of patients with peritoneal mesothelioma, and hyperthermia is known to enhance the effect of chemotherapy agents. ${ }^{30}$ Preliminary experiences in MPM have been rather disappointing, and survival with this technique does not seem to be better than following simple P/D. Severe complications such as permanent renal failure, bronchopleural fistula, postoperative empyema, and wound dehiscence have been associated with the use of hyperthermic intrapleural chemotherapy. ${ }^{17,18,31}$ Mortality has varied from nil to $11 \%$ following $P / D$ and hyperthermic intrapleural chemotherapy. ${ }^{17,18,31}$ Postoperative morbidity was $41 \%$ in a recent series. ${ }^{18}$ Median survival was 11 months in a Dutch series and 13 and 17 months in 2 American series. ${ }^{17,18,31}$

Povidone-iodine has been widely used as an antiseptic agent over the past 4 decades. Although mainly used for skin preparation prior to surgery, some have advocated its use in order to prevent tumoral seeding following resection of colorectal carcinoma. ${ }^{32,33}$ Povidone-iodine has a recognized antitumoral effect in colorectal carcinoma, breast carcinoma, and hepatoma. ${ }^{34}$ Opitz et al have shown that povidone-iodine has a direct cytotoxic effect on mesothelioma cells and induces necrosis of mesothelioma cells in vitro. ${ }^{35}$ Sun et al showed that diluted povidoneiodine inhibits tumor growth in a dose-dependent manner through apoptosis and suppression of superoxide dismutase activity in vitro. ${ }^{34}$ More recently, others have shown that exposure to increasing concentrations of povidone-iodine resulted in $100 \%$ lethality in mesothelioma cell cultures. ${ }^{36}$ For epithelioid and biphasic cell cultures, 10 minutes of exposure to $0.1 \%$ povidone-iodine was totally lethal, whereas $1 \%$ concentration and 10 minutes were necessary to kill all sarcomatoid cells. ${ }^{36}$ Interestingly, we have used a povidone-iodine concentration of $1 \%$ and an exposure time of minimum 15 minutes in all our patients. We cannot speculate on the impact of povidone-iodine lavage on patient's survival in our series. However, it is important to note that none of our patients had wound infection, or chest wall seeding. No patient developed clinical hypothyroidism in our series.

Considering the interesting effect of povidone-iodine, its wide availability, and the risks and complexity associated with intrapleural chemotherapy, we decided to design a protocol involving moderate hyperthermia, simple pleural lavage, and a moderate dose of povidone-iodine. We have been using povidone-iodine lavage for more than 15 years to induce pleurodesis following pleural abrasion in patients with recurrent spontaneous pneumothorax without any adverse side effects. Weder and his group have promoted the use of povidone-iodine-soaked towels in postpneumonectomy empyema and have reported no complication related to povidone-iodine. ${ }^{37}$ One publication reported cases of permanent blindness following intrapleural administration of povidone-iodine, but we believe this to be product-related rather than the povidone-iodine itself. ${ }^{38}$ Povidone-iodine has been used for many years to achieve pleurodesis in patients with pneumothorax or malignant pleural effusion. ${ }^{39}$ A recent meta-analysis including 499 
patients showed no major adverse effects. ${ }^{39}$ In the past, studies of intraperitoneal povidone-iodine have shown initial elevation of serum iodine following lavage but normal levels of iodine by 72 hours and no major changes in thyroxine levels. ${ }^{40}$

Although quality of life data were not collated, treatment acceptance and tolerance were good, and indeed $95 \%$ of patients received all 3 treatment modalities. We were somehow disappointed by the high local recurrence rate, and this is something we must concentrate on in the future. Recently, Friedberg et al reported encouraging results with intraoperative photodynamic therapy after $\mathrm{P} / \mathrm{D} .^{41} \mathrm{We}$ believe that immunotherapy or new drug therapies could be combined with $\mathrm{P} / \mathrm{D}$ or administered following P/D and help tackle residual microscopic disease and get better local control in the future.

We do recognize that our study suffers several limitations, including nonrandomization and patient selection bias based on prognostic factors. Over the past 10 years, approximately $50 \%$ of patients referred to our clinic were turned down for $\mathrm{P} / \mathrm{D}$ based on imaging (inoperable tumor, N3 or M1 disease), unfitness for surgery, or adverse prognostic factors. We used the Cancer and Leukemia Group $B$ predictive factors of survival to exclude patients unlikely to benefit from radical surgery. ${ }^{42}$ In addition, all surgical procedures were performed by a single surgeon (L.L.L.). This can be regarded as a limitation, because quality control is a potential problem in mesothelioma surgery and outcomes obtained at large-volume centers might not be reproduced in small-volume centers. ${ }^{43}$ The multimodality regimen used in this series is not standard: although P/D has been combined with systemic chemotherapy for more than 3 decades, the role of prophylactic radiotherapy after thoracotomy remains controversial. In addition, our cohort of patients is heterogeneous, with 14 patients receiving chemotherapy preoperatively, instead of adjuvant chemotherapy. However, we could not find any good reason to refuse surgery when patients were referred after completion of chemotherapy, and the tumor seemed completely resectable by P/D. Of note, 4 patients had preexisting empyema at the time of surgery, and this could have influenced their survival as only 1 was fit enough to receive chemotherapy postoperatively. The other 3 patients died within 5 to 16 months postoperatively. We are aware of the better prognosis of women with mesothelioma compared to men, with a threefold better survival. ${ }^{44}$ Our male-female ratio $(81: 21)$ is similar to the ones reported in other series and cannot have influenced positively survival in this series. We did not find that N1/N2 disease had a detrimental impact on survival, but this is likely to be related to small numbers as others have showed than N2 disease is an adverse prognostic factor in MPM. ${ }^{5,45}$ As others, we do believe that surgery should still be offered to mesothelioma patients who have completely resectable N2 disease. ${ }^{5,7}$ In this series, 5 of 26 patients $(19.2 \%)$ with N2 disease were still alive more than 3 years postoperatively. Because of multiple therapies being used in this study, the exact impact of povidone-iodine lavage on outcomes and longterm survival is not possible to precisely know. However, considering the body of evidence accumulated in vitro with povidone-iodine on mesothelioma cells, we believe this is a cheap, safe, and possibly useful adjunct after P/D. Despite having a majority of patients with stage 2 and 3 disease, our overall survival compares with the ones obtained in contemporary European series. ${ }^{25,46}$ A majority of patients relapsed following multimodality therapy, but remained fit enough to receive second-line or third-line therapies. This is something we did not have when using EPP, and we believe P/D advantageously preserves performance status, allowing patients to receive more therapies and live longer.

\section{CONCLUSIONS}

In our experience, $\mathrm{P} / \mathrm{D}$ with hyperthermic pleural lavage with povidone-iodine, prophylactic chest wall radiotherapy, and systemic chemotherapy is a safe and well-tolerated multimodality scheme. It is associated with low morbidity and mortality. This treatment plan represents an alternative to the classical trimodality regimen involving chemotherapy, EPP, and radiotherapy. Further research is needed to reduce local recurrence after P/D.

\section{References}

1. Peto J, Decarli A, La Vecchia C, Levi F, Negri E. The European mesothelioma epidemic. Br J Cancer. 1999;79:666-72.

2. Muers MF, Stevens RJ, Fisher P, et al. Active symptom control with or without chemotherapy in the treatment of patients with malignant pleural mesothelioma (MS01): a multicentre randomised trial. Lancet. 2008;371:1685-94.

3. Vogelzang NJ, Rusthoven JJ, Symanowski J, et al. Phase III study of pemetrexed in combination with cisplatin versus cisplatin alone in patients with malignant pleural mesothelioma. J Clin Oncol. 2003;21:2636-44.

4. Maziak DE, Gagliardi A, Haynes AE, Mackay JA, Evans WK. Surgical management of malignant pleural mesothelioma: a systematic review and evidence summary. Lung Cancer. 2005;48:157-69.

5. Sugarbaker DJ, Flores RM, Jacklitsch M, et al. Resection margins, extrapleural nodal status and cell type determine postoperative long-term survival in trimodality therapy of malignant pleural mesothelioma: results in 183 patients. $J$ Thorac Cardiovasc Surg. 1999;117:54-63.

6. Rusch VW, Piantadosi S, Holmes EC. The role of extrapleural pneumonectomy in malignant pleural mesothelioma. A Lung Cancer Study Group trial. J Thorac Cardiovasc Surg. 1991;102:1-9.

7. Krug LM, Pass HI, Rusch VW, et al. Multicenter phase II trial of neoadjuvant pemetrexed plus cisplatin followed by extrapleural pneumonectomy and radiation for malignant pleural mesothelioma. J Clin Oncol. 2009;27:3007-13.

8. Weder W, Stahel RA, Bernhard J, et al. Multicenter trial of neoadjuvant chemotherapy followed by extrapleural pneumonectomy in malignant pleural mesothelioma. Ann Oncol. 2007;18:1196-202.

9. De Perrot M, Feld R, Cho BC, et al. Trimodality therapy with induction chemotherapy followed by extrapleural pneumonectomy and adjuvant high-dose hemithoracic radiation for malignant pleural mesothelioma. J Clin Oncol. 2009;27: 1413-8.

10. Treasure T, Lang-Lazdunski L, Waller D, et al. Extra-pleural pneumonectomy versus no extra-pleural pneumonectomy for patients with malignant pleural mesothelioma: clinical outcomes of the mesothelioma and radical surgery (MARS) randomised feasibility study. Lancet Oncol. 2011;12:763-72. 
11. Sugarbaker DJ, Jaklitsch MT, Bueno R, et al. Prevention, early detection and management of complications after 328 consecutive extrapleural pneumonectomies. J Thorac Cardiovasc Surg. 2004;128:138-46.

12. Allen AM, Czerminska M, Janne P, et al. Fatal pneumonitis associated with intensity-modulated radiation therapy for mesothelioma. Int J Radiat Oncol Biol Phys. 2006;65:640-5.

13. Janne PA, Baldini EH. Patterns of failure following surgical resection for malignant pleural mesothelioma. Thorac Surg Clin. 2004;14:567-73.

14. Wanebo HJ, Martini N, Melamed MR, Hilaris B, Beattie EJ Jr. Pleural mesothelioma. Cancer. 1976;38:2481-8.

15. Neragi-Miandoab S, Richards WG, Sugarbaker DJ. Morbidity, mortality, mean survival and the impact of histology on survival after pleurectomy in 64 patients with malignant pleural mesothelioma. Int J Surg. 2008;6:293-7.

16. Lee TT, Everett DL, Shu HK, et al. Radical pleurectomy/decortication and intraoperative radiotherapy followed by conformal radiation with or without chemotherapy for malignant pleural mesothelioma. J Thorac Cardiovasc Surg. 2002; 124:1183-9.

17. Rusch V, Saltz L, Venkatraman E, et al. A phase II trial of pleurectomy/decortication followed by intrapleural and systemic chemotherapy for malignant pleural mesothelioma. J Clin Oncol. 1994;12:1156-63.

18. Richards WG, Zellos L, Bueno R, et al. Phase I to II study of pleurectomy/decortication and intraoperative intracavitary hyperthermic cisplatin lavage for mesothelioma. J Clin Oncol. 2006;24:1561-7.

19. Gupta V, Mychalczak B, Krug L, et al. Hemithoracic radiation after pleurectomy/ decortication for malignant pleural mesothelioma. Int J Radiat Oncol Biol Phys. 2005;63:1045-52.

20. Flores RM, Pass HI, Seshan VE, et al. Extrapleural pneumonectomy versus pleurectomy/decortication in the surgical management of malignant pleural mesothelioma: results in 663 patients. J Thorac Cardiovasc Surg. 2008;135: 620-6.

21. Boutin C, Rey F, Viallat JR. Prevention of malignant seeding after invasive diagnostic procedures in patients with pleural mesothelioma. Chest. 1995;108:754-8.

22. Butchart EG, Ashcroft T, Barnsley WC, Holden MP. Pleuropneumonectomy in the management of diffuse malignant mesothelioma of the pleura. Experience with 29 patients. Thorax. 1976;31:15-24.

23. Pilling JE, Nicholson AG, Harmer C, Goldstraw P. Prolonged survival due to spontaneous regression and surgical excision of malignant mesothelioma. Ann Thorac Surg. 2007;83:314-5.

24. Tilleman TR, Richards WG, Zellos L, et al. Extrapleural pneumonectomy followed by intracavitary intraoperative hyperthermic cisplatin with pharmacologic cytoprotection for treatment of malignant pleural mesothelioma: a phase 2 prospective study. J Thorac Cardiovasc Surg. 2009;138:405-11.

25. Minatel E, Trovo M, Polesel J, et al. Radical pleurectomy/decortication followed by high dose of radiation therapy for malignant pleural mesothelioma. Final results with long-term follow-up. Lung Cancer. 2014;83:78-82.

26. Bille A, Chicklore S, Okiror L, Cook GJ, Spicer J, Landau D, et al. Patterns of disease progression on ${ }^{18} \mathrm{~F}$-fluorodeoxyglucose positron-emission tomography computed tomography in patients with malignant mesothelioma undergoing multimodality therapy with pleurectomy/decortication. Nucl Med Commun. 2013; 34:1075-83.

27. Lang-Lazdunski L, Bille A, Lal R, et al. Pleurectomy/decortication is superior to extrapleural pneumonectomy in the multimodality management of patients with malignant pleural mesothelioma. J Thorac Oncol. 2012;7:737-43.

28. Broomfield S, Currie A, Van der Most RG, et al. Partial, but not complete, tumordebulking surgery promotes protective antitumor memory when combined with chemotherapy and adjuvant immunotherapy. Cancer Res. 2005;65:7580-4.

29. Predina JD, Kapoor V, Judy BF, et al. Cytoreduction surgery reduces systemic myeloid suppressor cell populations and restores intratumoral immunotherapy effectiveness. J Hematol Oncol. 2012;5:34.

30. Sugarbaker PH, Yan TD, Stuart OA, Yoo D. Comprehensive management of diffuse malignant peritoneal mesothelioma. Eur J Surg Oncol. 2006;32:686-91.

31. Van Ruth S, Baas P, Haas RL, Rutgers EJ, Verwaal VJ, Zoetmulder FA. Cytoreductive surgery combined with intraoperative hyperthermic intrathoracic chemotherapy for stage I malignant pleural mesothelioma. Ann Surg Oncol. 2003;10: 176-82.

32. Lee SW, Gleason NR, Bessler M, Whelan RL. Peritoneal irrigation with povidoneiodine solution after laparoscopic-assisted splenectomy significantly decreases port-tumor recurrence in a murine model. Dis Colon Rectum. 1999;42:319-26.

33. Balli JE, Franklin ME, Almeida JA, Glass JL, Diaz JA, Reymond M. How to prevent port-site metastases in laparoscopic colorectal surgery. Surg Endosc. 2000; 14:1034-6.
34. Sun P, Zhao JM, Luo ZC, et al. Diluted povidone-iodine inhibits tumor growth through apoptosis-induction and suppression of SOD activity. Oncol Rep 2012;27:383-8.

35. Opitz I, Sigrist B, Hillinger S, et al. Taurolidine and povidone-iodine induce different types of cell death in malignant pleural mesothelioma. Lung Cancer 2007;56:327-36

36. Fiorelli A, Pentimalli F, D'Urso V, et al. Antineoplastic activity of povidoneiodine on different mesothelioma cell lines: results of in vitro study. Eur J Cardiothorac Surg. 2014;45:993-1000.

37. Schneiter D, Grodzki T, Lardinois D, et al. Accelerated treatment of postpneumonectomy empyema: a binational long-term study. J Thorac Cardiovasc Surg. 2008; 136:179-85.

38. Wagenfeld L, Zeitz O, Richard G. Visual loss after povidone-iodine pleurodesis. N Engl J Med. 2007;357:1264-5.

39. Agarwal R, Khan A, Aggarwal AN, Gupta D. Efficacy and safety of iodopovidone pleurodesis: a systematic review and meta-analysis. Indian J Med Res 2012;135:297-303.

40. Sindelar WF, Mason GR. Intraperitoneal irrigation with povidone-iodine solution for the prevention of intra-abdominal abscesses in the bacterially contaminated abdomen. Surg Gynecol Obstet. 1979;148:409-11.

41. Friedberg JS, Culligan MJ, Mick R, et al. Radical pleurectomy and intraoperative photodynamic therapy for malignant pleural mesothelioma. Ann Thorac Surg. 2012;93:1658-65.

42. Herndon JE, Green MR, Chahinian AP, Corson JM, Suzuki Y, Vogelzang NJ Factors predictive of survival among 337 patients with mesothelioma treated between 1984 and 1994 by the Cancer and Leukemia Group B. Chest. 1998;113: 723-31.

43. Burt BM, Cameron RB, Mollberg NM, et al. Malignant pleural mesothelioma and the Society of Thoracic Surgeons database: an analysis of surgical morbidity and mortality. J Thorac Cardiovasc Surg. 2014;148:30-5.

44. Taioli E, Wolf AS, Camacho-Rivera M, Flores RM. Women with malignant pleural mesothelioma have a threefold better survival rate than men. Ann Thorac Surg. 2014;98:1020-4.

45. Rusch VW, Giroux D, Kennedy C, et al. Initial analysis of the international association for the study of lung cancer mesothelioma database. J Thorac Oncol. 2012; 7:1631-9.

46. Bolukbas S, Manegold C, Eberlein M, et al. Survival after trimodality therapy for malignant pleural mesothelioma: radical pleurectomy, chemotherapy with cisplatin/pemetrexed and radiotherapy. Lung Cancer. 2011;71:75-81.

\section{Discussion}

Dr M. de Perrot (Toronto, Ontario, Canada). Dr Weder was going to be the discussant but he couldn't come this morning, so I will discuss the paper for him.

Congratulations for your nice work. Over the past 10 years, you treated 98 patients with different stages and histology that were treated with that protocol.

I have 4 short questions. How many patients with malignant pleural mesothelioma that you evaluated were selected finally for this treatment?

Dr Lang-Lazdunski. At the moment, it is roughly $50 \%$ of patients that I operate, but with time, because of the center effect, we have been referred more and more patients, and I think our oncologists, our chest physicians in the United Kingdom were initially a bit shy to refer patients, but looking at the results we have now, we have more referrals. Roughly, I would say I operate 1 out of $2 \mathrm{pa}-$ tients I am referred.

Dr de Perrot. Although you had planned for adjuvant chemotherapy, you still had a number of patients who had neoadjuvant chemotherapy before the procedure?

Dr Lang-Lazdunski. It wasn't really neoadjuvant. Thirteen patients had chemotherapy because surgery was not going to be an option, and then either the patient asked to be referred to a 
surgical center, or, because of a good response, the oncologist decided that the patient should be referred for surgery and I found there was no reason to say no. So I took patients sometimes 3 months after chemotherapy, sometimes 1 year after chemotherapy.

Dr de Perrot. From the radiation perspective, the Boutin study with adjuvant radiation on the port site was not confirmed in subsequent randomized trials. Do you feel that it is necessary to give radiation therapy, and would you consider extending the radiation field to an intrathoracic site where gross disease was left or where there was more risk of disease progression?

Dr Lang-Lazdunski. There is still controversy about radiotherapy, and there is a trial at the moment going on in the United Kingdom enrolling more than 300 patients to find out. I am a believer in this technique. The fact is, whether it's radiotherapy or the Betadine, we have not had any patients developing chest wall implant at 1 year in this study. What I must say is that I very often use 2 access incisions, 1 in the fifth interspace and 1 in the eighth interspace, whether it is to clear or replace the diaphragm, and I always insist with the radiation oncologists that all of those incisions are included in the radiation field, which is very important, as well as at previous port sites, because most of the patients had a previous video-assisted thoracoscopic surgery procedure. I will just make 1 comment. Having a talc pleurodesis before the operation facilitates the procedure. It is never a contraindication. It makes your operation easier.

Dr de Perrot. Finally, 42\% had an R2 resection. In the future, what do you think you could improve to decrease that number-a more extensive surgery? Do you think extrapleural pneumonectomy still has a role for the patient with R2 disease?

Dr Lang-Lazdunski. As you know, I dropped extrapleural pneumonectomy in 2008. I think patients are happier with this procedure, there is better quality of life, and I showed in a previous paper when I compared my results that survival was not dissimilar between those who had an extrapleural pneumonectomy (EPP) or an incomplete macroscopic resection. So I don't think that switching to EPP adds any benefit. Certainly it doesn't add any quality of life and it doesn't add to survival, in our experience at least.

Dr de Perrot. Did you see a decrease in the number of R2 resections over time, over the 10 years?

Dr Lang-Lazdunski. Yes. I'm still learning.

Dr de Perrot. Thank you.

Dr Lang-Lazdunski. Thank you, Marc.

Dr D. Miller (Marietta, Ga). Excellent presentation.

When we have done pleurectomy and decortication, the recurrences that we had were related to the pericardium, and so when we do this now, we take out the pericardium also and do reconstruction. From your experience, how do you handle the pericardium and pericardial disease?

Dr Lang-Lazdunski. I start this operation exactly like an EPP. I basically dissect the extrapleural plane. I take all the anterior mediastinal fat together with the mediastinal pleura. Sometimes it is quite sporty with the phrenic nerve, but I try to save the pericardium if I have a good dissection plan because I think it is a great natural barrier, just like the diaphragm. In those with through-andthrough nodules, obviously you have no option and I do resect the pericardium and replace it. We do have pericardial relapse. It has not been the main problem in our study. Most relapses are on the diaphragm.

Dr J. Friedberg (Philadelphia, $\mathrm{Pa}$ ). With respect to the pericardium, I would just say that since you are leaving the lung, I just take the pericardium out if it is involved and leave it, I don't reconstruct it, and I haven't had a problem with that.

Loic, I congratulate you on this study. As we are doing lungsparing surgery, the greatest variable is the surgeon, so it is incredibly valuable to have 1 surgeon doing the operation; this allows you to evaluate the intraoperative adjuvant better than any other assessment. Did you have any cohort of patients who for some reason did not receive the Betadine, and how did they do in comparison? Are you just that good a surgeon and they are getting these great results or do you think the Betadine is contributing?

Dr Lang-Lazdunski. To be honest, I always go for the Betadine lavage. In the last 10 years, I have always used it. It is an extra 20 minutes. It is very simple. There is no proper internal control, but my colleagues in the unit do a slightly different procedure. I'm not sure they always go for a total pleurectomy, and they don't use Betadine, and their results are very similar to the ones we had with EPP before. Clearly, the survival in my experience is way, way better.

Dr Friedberg. I have 2 observations, and I would hope when you write the paper that you would comment on these. You seem to have a higher proportion of lower-stage patients, than you normally see in a surgical series. They all seem to get upstaged. I was wondering about that.

The other thing I would hope you would comment on is why the nodal disease did not make a difference in your outcomes, if I saw that slide correctly.

Dr Lang-Lazdunski. I think it is a small series and that is why the nodal disease didn't impact on survival. I have discussed this issue with many in the audience already. I have patients with nonbulky N2 disease still alive at 4 years or sometimes 5 years, especially epithelioid patients. It is a different beast from lung cancer. As long as you can resect the N2, I don't think N2 should be a contraindication to surgery.

Dr Friedberg. And my last comment with respect to it being a different beast than lung cancer is that I think we need to stop thinking about the survival in this disease with an eye toward local recurrence being the endpoint. The series from MD Anderson had a $90 \%$ local control rate with extrapleural pneumonectomy and radiation and had, if I recall, a 14-month median survival. Here you have a median survival in the 40-month range and with the local recurrence as the predominant location. So I do think that, as a group, we need to start thinking about what our endpoint is. Maybe it is somehow promoting the disease-free survival, understanding that we are going to have a local recurrence.

Dr Lang-Lazdunski. We have used the CyberKnife successfully for very focal relapse. I have reoperated 2 patients. I know that Dr David Sugarbaker and his group do reoperate more liberally. I think maybe we will have a clue once Dr Lee Krug has finished his phase 2 trial with adjuvant pleural-type radiotherapy, which is very exciting, and there are so many targeted agents now like anti-PD1 and other immune checkpoint inhibitors, that the future looks bright really for these patients.

Dr Friedberg. It's great work. Thank you. 